\title{
n-PRÜFER DOMAINS
}

\author{
SANg Bum LeE
}

\begin{abstract}
We introduce $n$-Prüfer domains which are generalisations of Prüfer domains and give several characterisations of them in terms of generalisations of purity, flatness and absolute purity.
\end{abstract}

\section{INTRODUCTION}

Let $R$ be a commutative domain with 1 and $Q$ its field of quotients. $R$ is called Prüfer if every finitely generated ideal of $R$ is projective. Prüfer domains have been characterised in numerous ways. Classical results can be found in Gilmer [3].

Here we wish to introduce a generalisation of Prüfer domains: we shall call a domain $R n$-Prüfer (for integers $n>0$ or $n=\infty$ ) if every finitely generated torsion-free $R$-module of projective dimension $\leqslant n-1$ is projective. Note that every domain is 1-Prüfer and the $\infty$-Prüfer domains are exactly the Prüfer domains. Trivially, Prüfer domains are $n$-Prüfer for every $0<n<\infty$, but the converse does not hold.

Examples of such domains $R$ which are $n$-Prüfer for every $0<n<\infty$, but not Prüfer, are Noetherian domains of Krull dimension 1 which are not integrally closed. The following argument verifies the claim. Let $M$ be a finitely generated, torsion-free $R$-module of projective dimension $\leqslant 1$. Embedding of $M$ into a finitely generated free $R$-module $F$ yields the $R$-module $F / M$. If the projective dimension of $M$ is 1 , then the projective dimension of $F / M$ is 2 , which contradicts the fact that the Krull dimension of Noetherian domain is equal to its finitistic projective dimension (see, Raynaud and Gruson [8]). Hence $M$ is projective, and thus $R$ is 2-Prüfer. Now, induction on the projective dimension of $M$ establishes our claim.

In contrast, Noetherian domains $R$ of Krull dimension $>1$ are 1-Prüfer but not 2-Prüfer (and thus not $n$-Prüfer for any $n \geqslant 2$ ). Indeed, let $n>1$ be the Krull dimension of $R$. By the definition of finitistic projective dimension, there exists an ideal of $R$ of projective dimension $n-1$. Hence $R$ is not $n$-Prüfer. Now the claim follows from the

\section{Received 15th April, 2003}

The paper was completed while the author was visiting Tulane University, and expresses his thanks to the Department of Mathematics of Tulane University for the hospitality and also Professor Laszlo Fuchs for several discussions on the subject.

Copyright Clearance Centre, Inc. Serial-fee code: 0004-9727/03 \$A2.00+0.00. 
fact that coherent 2-Prüfer domains are $n$-Prüfer every $2 \leqslant n<\infty$, which can be easily proved by induction argument.

In this note, we wish to prove that $n$-Prüfer domains have a number of characterisations, in particular, in terms of generalisations of purity, flatness and absolute purity (see Corollary 2). We also generalise a result on Prüfer domains concerning the coherency of polynomial rings to $n$-Prüfer domains (see Theorem 2). Throughout this note, $n$ will be a positive integer or $\infty$. For unexplained definitions and terminologies, we refer to Fuchs and Salce $[1,2]$ and Rotman [7].

\section{Preliminaries}

Recall that an $R$-module $M$ is flat if $\operatorname{Tor}_{1}^{R}(M, N)=0$ holds for all finitely presented $R$-modules $N$. The following generalisation of flatness will be used. An $R$-module $M$ will be called $n$-fiat if $\operatorname{Tor}_{1}^{R}(M, N)=0$ holds for all finitely presented $R$-modules $N$ with projective dimension $\leqslant n$. Obviously, direct sums and summands of $n$-flat modules are again $n$-flat. An $R$-submodule $N$ of $M$ is said to be relatively divisible (or briefly $R D$ ) in $M$ if $r N=N \cap r M$ for each $r \in R$. Accordingly, an exact sequence $0 \rightarrow N \rightarrow M \rightarrow L \rightarrow 0$ is called an $R D$-exact sequence if the inclusion map embeds $N$ in $M$ as an $R D$-submodule.

We start our discussion with a lemma.

Lemma 1. An $R$-module $M$ is 1-flat if and only if it is torsion-free.

Proof Let $E$ be an injective cogenerator of the category of $R$-modules, and suppose that the $R$-module $M$ is 1-flat, that is, it satisfies $\operatorname{Tor}_{1}^{R}(N, M)=0$ for all finitely presented $R$-modules $N$ with projective dimension $\leqslant 1$. From the natural isomorphism

$$
\operatorname{Ext}_{R}^{1}\left(N, \operatorname{Hom}_{R}(M, E)\right) \cong \operatorname{Hom}_{R}\left(\operatorname{Tor}_{1}^{R}(N, M), E\right),
$$

it follows that $\operatorname{Ext}_{R}^{1}\left(N, \operatorname{Hom}_{R}(M, E)\right)=0$ for all finitely presented $R$-modules $N$ with projective dimension $\leqslant 1$. By Fuchs and Salce $\left[1\right.$, p. 36], $\operatorname{Hom}_{R}(M, E)$ is then a divisible $R$-module. For the torsion submodule $t M$ of $M$ we have the $R D$-exact sequence $0 \rightarrow$ $t M \rightarrow M \rightarrow M / t M \rightarrow 0$ which induces the $R D$-exact sequence $0 \rightarrow \operatorname{Hom}_{R}(M / t M, E) \rightarrow$ $\operatorname{Hom}_{R}(M, E) \rightarrow \operatorname{Hom}_{R}(t M, E) \rightarrow 0$. Here $\operatorname{Hom}_{R}(t M, E)$ is divisible as an epic image of $\operatorname{Hom}_{R}(M, E)$ and reduced (because $t M$ is torsion), thus it is 0 . Hence $t M$ is 0 , and $M$ is torsion-free.

Conversely, if $M$ is a torsion-free $R$-module, then the injection map $M \rightarrow Q \otimes M$ induces an epimorphism $\operatorname{Hom}_{R}\left(Q \bigotimes_{R} M, E\right) \rightarrow \operatorname{Hom}_{R}(M, E)$. Since $Q \bigotimes_{R} M$ is torsionfree, the $R$-module $\operatorname{Hom}_{R}(Q \underset{R}{\otimes} M, E)$ is $h$-divisible (that is, an epic image of an injective $R$-module), and therefore so is $\operatorname{Hom}_{R}(M, E)$. From the exact sequence 0 $\rightarrow H \rightarrow D \rightarrow \operatorname{Hom}_{R}(M, E) \rightarrow 0$, where $D$ is a direct sum of copies of $Q$, we obtain $\operatorname{Ext}_{R}^{1}\left(N, \operatorname{Hom}_{R}(M, E)\right) \cong \operatorname{Ext}_{R}^{2}(N, H)$. The second Ext is 0 whenever projective $\operatorname{dimension}_{R} N \leqslant 1$, so the same holds for the first Ext. Hence $\operatorname{Hom}_{R}\left(\operatorname{Tor}_{1}^{R}(N\right.$, 
$M), E)=0$ follows. By the choice of $E$ we conclude $\operatorname{Tor}_{1}^{R}(N, M)=0$, showing that $M$ is 1-flat.

Recall that an $R$-module $D$ is said to be absolutely pure (or FP-injective) if it is a pure submodule in every $R$-module containing it as a submodule. Megibben [6] proved that an $R$-module $D$ is absolutely pure if and only if $\operatorname{Ext}_{R}^{1}(N, D)=0$ for all finitely presented $R$-modules $N$. Accordingly, we define an $R$-module $D$ to be $n$-absolutely pure if, for all finitely presented $R$-modules $N$ of projective dimension $\leqslant n$, we have $\operatorname{Ext}_{R}^{1}(N, D)=0$. It follows at once that direct sums and summands of $n$-absolutely pure modules are again $n$-absolutely pure. A result similar to Lemma 1 can be obtained.

LEMma 2. An $R$-module $D$ is 1-absolutely pure if and only if it is divisible

Proof: If $D$ is 1-absolutely pure $R$-module, then $\operatorname{Ext}_{R}^{1}(R / L, D)=0$ for all projective ideals $L$. By Fuchs and Salce $[1$, p. 36], this amounts to the divisibility of $D$. Conversely, suppose $D$ is a divisible $R$-module, and $N$ is a finitely presented $R$-module of projective dimension $\leqslant 1$. Since $N$ has a finite projective resolution, we can apply the natural isomorphism (see for example, Rotman [7, p. 257])

$$
\operatorname{Tor}_{1}^{R}\left(\operatorname{Hom}_{R}(D, E), N\right) \cong \operatorname{Hom}_{R}\left(\operatorname{Ext}_{R}^{1}(N, D), E\right)
$$

for an injective cogenerator $E$. Here $\operatorname{Hom}_{R}(D, E)$ is a torsion-free $R$-module, so by Lemma 1, it is 1-flat. This implies that the Tor in the last formula vanishes, and consequently, the right side is 0 . This leads to the equation $\operatorname{Ext}_{R}^{1}(N, D)=0$, which amounts the 1-absolute purity of $D$.

\section{3. $n$-PURITY}

Recall that an $R$-module $A$ of $B$ is said to be pure if, for all (finitely presented) $R$ modules $N$, the map $N \bigotimes_{R} A \rightarrow N \bigotimes_{R} B$ induced by the inclusion $A \rightarrow B$ is injective. In the same spirit as flatness and absolute purity were generalised, we define a generalisation: an $R$-submodule $A$ of $B$ is called $n$-pure if, for all finitely presented $R$-modules $N$ of projective dimension $\leqslant n$, the map $N \bigotimes_{R} A \rightarrow N \bigotimes_{R} B$ induced by the inclusion $A \rightarrow B$ is injective, or equivalently, the $\operatorname{map} \operatorname{Hom}_{R}(N, B) \rightarrow \operatorname{Hom}_{R}(N, B / A)$ induced by the natural map $B \rightarrow B / A$ is surjective.

Lemma 3. For every $n(0<n \leqslant \infty)$, the following conditions on an $R$-module $D$ are equivalent:

(a) $D$ is $n$-absolutely pure;

(b) $D$ is $n$-pure in any (injective) $R$-module $E$ containing $D$ as an $R$ submodule;

(c) each $R$-homomorphism $\phi: H \rightarrow D$ from a finitely generated $R$-submodule $H$ of projective dimension $\leqslant n-1$ of a finitely generated free $R$-module $F$ is induced by a map $\gamma: F \rightarrow D$. 
Proof (a) $\Leftrightarrow$ (b) Consider an exact sequence $0 \rightarrow D \rightarrow E \rightarrow E / D \rightarrow 0$ where $E$ is an injective $R$-module containing $D$. Let $N$ be a finitely presented $R$-module of projective dimension $\leqslant n$. The induced sequence $0 \rightarrow \operatorname{Hom}_{R}(N, D) \rightarrow \operatorname{Hom}_{R}(N, E)$ $\rightarrow \operatorname{Hom}_{R}(N, E / D) \rightarrow \operatorname{Ext}_{R}^{1}(N, D) \rightarrow \operatorname{Ext}_{R}^{1}(N, E)=0$ establishes the result.

(a) $\Leftrightarrow$ (c) The exact sequence $0 \rightarrow H \rightarrow F \rightarrow F / H \rightarrow 0$ along with the induced sequence $0 \rightarrow \operatorname{Hom}_{R}(F / H, D) \rightarrow \operatorname{Hom}_{R}(F, D) \rightarrow \operatorname{Hom}_{R}(H, D) \rightarrow \operatorname{Ext}_{R}^{1}(F / H, D)$ $\rightarrow \operatorname{Ext}_{R}^{1}(F, D)=0$ implies the result, since $F / H$ is finitely presented of projective dimension $\leqslant n$.

Note that for an $R$-submodule $H$ of a flat $R$-module $F, H$ is pure in $F$ if and only if $F / H$ is flat. This can be generalised easily.

Lemma 4. Let $H$ be an $R$-submodule of an n-flat module $F$. Then $H$ is n-pure in $F$ if and only if $F / H$ is n-flat.

In particular, we have

COROLlary 1 . For an $R$-submodule $A$ of a torsion-free $R$-module $B$, the following are equivalent:

(a) $A$ is relatively divisible in $B$;

(b) $A$ is 1-pure in $B$;

(c) $B / A$ is torsion-free.

Note that $n$-purity can also be characterised in the following way. Consider finite systems of equations over $N$,

$$
\sum_{j=1}^{l} r_{i j} x_{j}=a_{i} \in N \quad(i=1, \ldots, k),
$$

where $r_{i j} \in R$ and $x_{1}, \ldots, x_{l}$ are unknowns such that the $R$-submodule $H$ of the free $R$ module $F$ on $\left\{x_{1}, \ldots, x_{l}\right\}$ generated by the left members of (1) is of projective dimension $\leqslant n-1 . \quad N$ is $n$-pure in $M$ if and only if every such system (1) has a solution in $N$ whenever it has a solution in $M$. Such a system (1) will be called an $n$-finite system. Note that an $n$-finite system is an $m$-finite system whenever $n \leqslant m$.

LEMma 5. Let $L \leqslant N \leqslant M$ be $R$-modules.

(a) If $L$ is $n$-pure in $M$, then it is also n-pure in $N$.

(b) If $L$ is $n$-pure in $N$ and $N$ is $n$-pure in $M$, then $L$ is $n$-pure in $M$.

(c) If $N$ is $n$-pure in $M$, then $N / L$ is $n$-pure in $M / L$. The converse holds if $L$ is $n$-pure in $M$.

Proof: (a) Any $n$-finite system of equations over $L$ which is solvable in $N$ is trivially solvable in $M$. Hence it has a solution in $L$ by hypothesis.

(b) Any $n$-finite system of equations over $L$ which is solvable in $M$ can be viewed as a system over $N$. Since $N$ is $n$-pure in $M$, it has a solution in $N$. Again, since $L$ is $n$-pure in $N$, it has a solution in $L$. 
(c) Suppose $\sum_{j=1}^{l} r_{i j} x_{j}=a_{i}+L \in N / L$ is an $n$-finite system of equations over $N / L$ which has a solution $x_{j}=b_{j}+L \in M / L$, where $1 \leqslant i \leqslant k, 1 \leqslant j \leqslant l$. Then $\sum_{j=1}^{l} r_{i j} b_{j}$ $=a_{i}+p_{i}$ for some $p_{i} \in L$. Hence $\sum_{j=1}^{l} r_{i j} x_{j}=a_{i}+p_{i} \in N$ is an $n$-finite system of equations over $N$ which has a solution $x_{j} \stackrel{j=1}{=} b_{j} \in M(1 \leqslant j \leqslant l)$. Then $\sum_{j=1}^{l} r_{i j}\left(b_{j}+L\right)=a_{i}+L$ and $x_{j}=b_{j}+L \in M / L$ is a solution of the original $n$-finite system. To prove the converse, let $U$ be a finitely presented $R$-module of projective dimension $\leqslant n$. Then we have a commutative digram

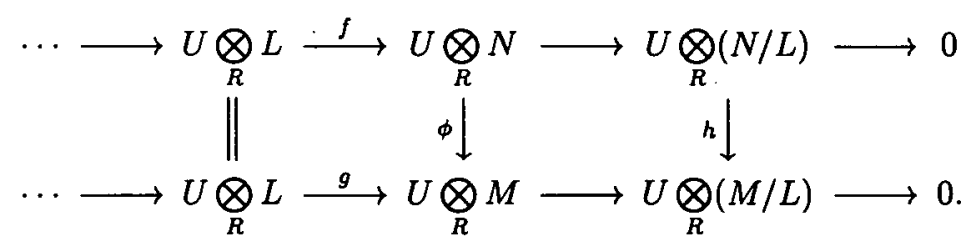

Since $L$ is $n$-pure in $M$ and thus $L$ is $n$-pure in $N$ by (a), we have monomorphisms $f, g$. Since $h$ is a monomorphism by hypothesis, $\phi$ is a monomorphism, too.

We take a self-evident definition of an $n$-pure-exact sequence similar to that of an $R D$-exact sequence. By Lemma 5 , those elements of $\operatorname{Ext}_{R}^{1}(N, M)$ which are represented by $n$-pure-exact sequence form a subgroup, which will be denoted by $n$ - $\operatorname{Pext}_{R}^{1}(N, M)$. It is readily checked that $n$ - $\operatorname{Pext}_{R}^{1}(N, M)=0$ for all finitely presented modules $N$ of projective dimension $\leqslant n$ and for all $R$-modules $M$.

\section{4. $n$-PRÜFER DOMAINS}

For $n$-Prüfer domains we can now prove our main result.

THEOREM 1. For a domain $R$ and for every $n(0<n \leqslant \infty)$, the following conditions are equivalent:

(a) $R$ is $n$-Prüfer;

(b) 1 -absolutely pure $R$-modules are $n$-absolutely pure;

(c) 1-pure $R$-submodules of all $R$-modules are $n$-pure.

Proof (c) $\Rightarrow$ (b) Let $D$ be a 1-absolutely pure $R$-module. By Lemma $3, D$ is 1-pure in its injective hull $E(D)$. By hypothesis, it is $n$-pure, which implies that $D$ is $n$-absolutely pure.

(b) $\Rightarrow$ (a) Let $N$ be a finitely generated torsion-free $R$-module of projective dimension $\leqslant n-1$. Imbed $N$ into a finitely generated free $R$-module $F$. Then $F / N$ is a finitely presented $R$-module of projective dimension $\leqslant n$. Therefore, by hypothesis, $\operatorname{Ext}_{R}^{1}(F / N, D)=0$ for all divisible $R$-modules $D$. By Lee [5], projective dimension of $F / N$ is $\leqslant 1$, which implies that $N$ is projective. 
(a) $\Rightarrow$ (c) Let $A$ be a 1-pure $R$-submodule of $B$ and $N$ a finitely presented $R$-module of projective dimension $\leqslant n$. Consider a presentation $0 \rightarrow H \rightarrow F \rightarrow N \rightarrow 0$ of $N$ where $H$ is a finitely generated torsion-free $R$-module of projective dimension $\leqslant n-1$. By hypothesis, $H$ is projective and thus $N$ has projective dimension $\leqslant 1$. Since $A$ is 1-pure, the map $A \bigotimes_{R} N \rightarrow B \bigotimes_{R} N$ induced by the inclusion $A \rightarrow B$ is injective. We conclude that $A$ is $n$-pure.

Lemma 6. 1-flat $R$-modules over $n$-Prüfer domains $R$ are $n$-flat.

Proof: Let $A$ be a 1 -flat $R$-module and $N$ be a finitely presented $R$-module of projective dimension $\leqslant n$. In the natural isomorphism

$$
\operatorname{Ext}_{R}^{1}\left(N, \operatorname{Hom}_{R}(A, E)\right) \cong \operatorname{Hom}_{R}\left(\operatorname{Tor}_{1}^{R}(N, A), E\right)
$$

where $E$ is an injective $R$-module, Ext is 0 since $\operatorname{Hom}_{R}(A, E)$ is divisible and thus $n$-sbsolutely pure by hypothesis. Hence the right Hom is 0 . Since $E$ was arbitary, $\operatorname{Tor}_{1}^{R}(N, A)=0$, which implies that $A$ is $n$-flat.

In Lee [4], a domain $R$ was called $n$-coherent if every finitely generated torsion-free $R$-module of projective dimension $\leqslant n-1$ is finitely presented. $n$-Prüfer domains are trivially $n$-coherent, and we are going to show that the converse holds when 1-flat modules are $n$-flat.

Lemma 7. For a domain $R$ and for every $n(0<n \leqslant \infty)$, the following are equivalent:

(a) $R$ is $n$-Prüfer;

(b) $R$ is $n$-coherent and all 1-flat $R$-modules are $n$-flat.

Proof: In view of Lemma 6, we have only to prove (b) implies (a). Let $D$ be a 1-absolutely pure $R$-module and $N$ a finitely presented $R$-module of projective dimension $\leqslant n$. Since $R$ is $n$-coherent, $N$ has a finite projective resolution (see Lee [4]). In the natural isomorphism

$$
\operatorname{Tor}_{1}^{R}\left(N, \operatorname{Hom}_{R}(D, E)\right) \cong \operatorname{Hom}_{R}\left(\operatorname{Ext}_{R}^{1}(N, D), E\right),
$$

where $E$ is an injective $R$-module, Tor is 0 since $\operatorname{Hom}_{R}(D, E)$ is torsion-free and thus $n$-flat by hypothesis. Hence $\operatorname{Ext}_{R}^{1}(N, D)=0$ by the choice of $E$. This implies that $D$ is $n$-absolutely pure. By Theorem $1, R$ is $n$-Prüfer.

It is proved in Fuchs and Salce [2, p. 247] that if a domain $R$ is Prüfer, then the torsion $R$-submodule of every $R$-module is pure. Now we can generalise this result.

Lemma 8. Over $n$-Prüfer domains $R$, the torsion $R$-submodule of every $R$-module is $n$-pure.

Proof. Let $t(M)$ be the torsion $R$-submodule of an $R$-module $M$. Then $M / t(M)$ is torsion-free and thus $n$-flat by Lemma 7 . Hence $\operatorname{Tor}_{1}^{R}(N, M / t(M))=0$ for all finitely 
presented $R$-module $N$ of projective dimension $\leqslant n$. Therefore the map $t(M) \bigotimes_{R} N$ $\rightarrow M \bigotimes_{R} N$ induced by the inclusion $t(M) \rightarrow M$ is injective. This implies that $t(M)$ is $n$-pure in $M$.

We quote a result by Sabbagh [9] which we want to generalise.

LEMMA 9. (Sabbagh [9].) The ring of polynomials in an arbitrary number of variables over a Prüfer domain is coherent.

THEOREM 2. If $R$ is $n$-Prüfer, then $R\left[x_{1}, \ldots, x_{k}\right]$ is $n$-coherent for every integers $k \geqslant 1$.

Proof: Let $M$ be a finitely generated torsion-free $R\left[x_{1}, \ldots, x_{k}\right]$-module with projective dimension ${ }_{R\left[x_{1}, \ldots, x_{k}\right]} M \leqslant n-1$. Since $R\left[x_{1}, \ldots, x_{k}\right]$ is a free $R$-module, $M$ is a finitely generated torsion-free $R$-module with projective dimension ${ }_{R} M \leqslant n-1$. By hypothesis, $M$ is a projective $R$-module and therefore flat. By Raynaud and Gruson [8, Theorem 3.4.6], $M$ is a finitely presented $R\left[x_{1}, \ldots, x_{k}\right]$-module. This implies that $R\left[x_{1}, \ldots, x_{k}\right]$ is $n$-coherent.

We can also verify:

Lemma 10. If $R_{P}$ is $n$-Prüfer for every maximal ideal $P$ of $R$, then $R$ is $n$-Prüfer.

Proof: Suppose $M$ is a finitely generated torsion-free $R$-module with projective dimension $_{R} M \leqslant n-1$. Then $M_{P}$ is a finitely generated torsion-free $R_{P-}$ module with projective dimension $R_{P} M_{P} \leqslant n-1$. By hypothesis, $M_{P}$ is a projective $R_{P}$-module. By Fuchs and Salce [2, p. 196], $M$ is a projective $R$-module. Hence $R$ is $n$-Prüfer.

Combining all these, we have

COROLlaRY 2 For a domain $R$ and for every $n(0<n \leqslant \infty)$, the following implications hold:

$$
\text { (a) } \Rightarrow \text { (b) } \Leftrightarrow \text { (c) } \Leftrightarrow \text { (d) } \Leftrightarrow \text { (e) } \Rightarrow \text { (f) } \Rightarrow \text { (g) , }
$$

where
(a) $R_{P}$ is $n$-Prüfer for every ideal $P$ of $R$.
(b) $R$ is $n$-Prüfer.
(c) 1-purity implies n-purity.
(d) 1-absolute purity implies $n$-absolute purity.
(e) $R$ is $n$-coherent and 1-flatness implies $n$-flatness.
(f) 1-flatness implies $n$-flatness.
(g) In every $R$-module the torsion submodule is $n$-pure.

\section{REFERENCES}

[1] L. Fuchs and L. Salce, Modules over valuation domains, Lecture Notes in pure and Applied Math. 97 (Marcel Dekker, New York, 1984). 
[2] L. Fuchs and L. Salce, Modules over non-Noetherian domains, Math. Surveys and Monographs 84 (Amer. Math. Society, Providence, R.I., 2001).

[3] R. Gilmer, Multiplicative ideal theory, Queens Papers Pure Appl. Math. 90 (Queens University, Kingston, ON, 1972).

[4] S.B. Lee, ' $n$-coherent rings', Comm. Algebra 30 (2002), 1119-1126.

[5] S.B. Lee, 'Semi-Baer modules over domains', Bull. Austral. Math. Soc. 64 (2001), 21-26.

[6] C. Megibben, 'Absolutely pure modules', Proc. Amer. Math. Soc. 26 (1970), 561-566.

[7] J. Rotman, An introduction to homological algebra (Academic Press, New York, 1979).

[8] M. Raynaud and L. Gruson, 'Critères de platitude et de projectivitè techniques de "platification" d'un module', Invent. Math. 13 (1971), 1-89.

[9] G. Sabbagh, 'Coherence of polynomial rings and bounds in polynomial ideals', J. Algebra 31 (1974), 499-507.

Department of Mathematical Education

Sangmyung University

Seoul 110-743

Korea

e-mail: sblee@sangmyung.ac.kr 